\title{
Impact of different doses and application methods of biophos (Biofertilizer) on growth and fodder yield of maize
}

Khalil Ahmed Brohi ${ }^{1}$, Abdul Haleem Sial ${ }^{1}$, Ayaz Latif Siyal ${ }^{2 *}$, Kamran Razzaque Kalwar ${ }^{1}$, Babar Sultan Mengal ${ }^{1}$, Fateh Muhammad Baloch ${ }^{3}$, Aneel Ahmed Korejo ${ }^{1}$ and Manthar Ali Sahito ${ }^{1}$

1. Department of Agronomy, Faculty of Crop Production, Sindh Agriculture University Tandojam, Pakistan

2. Department of Plant Breeding and Genetics, Sindh Agriculture University Tandojam, Sindh, Pakistan

3. Social Science Institute, Agriculture Research Institute, Quetta (PARC), Pakistan

*Corresponding author's email:Latifayazsiyal@gmail.com

Citation

Khalil Ahmed Brohi, Abdul Haleem Sial, Ayaz Latif Siyal, Kamran RazzaqueKalwar, Babar Sultan Mengal, Fateh Muhammad Baloch, Aneel Ahmed Korejo and Manthar Ali Sahito. Impact of different doses and application methods of biophos (Biofertilizer) on growth and fodder yield of maize. Pure and Applied Biology. Vol. 11, Issue 3, pp725733. http://dx.doi.org/10.19045/bspab.2022.110073

Received: 21/05/2021 Revised: 26/06/2021

Accepted: 19/10/2021

Online First: 04/11/2021

\section{Abstract}

The increased reliance of novel agriculture on extreme artificial input of chemical fertilizers has caused numerous environmental problems related to greenhouse effect, soil deterioration, and air and water pollution. A field study was carried out at Sindh Agriculture University, Tandojam during Kharif 2013. The analysis of variance illustrated that different doses and application methods of Biophos (Biofertilizer) as seed treatment before sowing or broadcasting after sowing affected positively and significantly $(\mathrm{P}<0.05)$ seed germination, growth and fodder yield traits of maize variety Hycorn-8288 as compared to No Biophos (Control). Combination of Biophos $10 \mathrm{~g} \mathrm{~kg}^{-1}$ seed (Seed treatment before sowing) + Biophos $25.0 \mathrm{~kg} \mathrm{ha}^{-1}$ (Broadcasting) resulted in maximum (33.00 m $\mathrm{m}^{-2}$ ) seed germination, plant height $(178.87 \mathrm{~cm})$, leaves plant ${ }^{-1}(14.80)$, nodes plant ${ }^{-1}(5.40)$, stem girth $(4.75 \mathrm{~cm})$, fodder weight $\left(4.13 \mathrm{~kg} \mathrm{~m}^{-2}\right)$ and fodder yield $\left(41.33 \mathrm{t} \mathrm{ha}^{-1}\right)$, whereas combination of Biophos $10 \mathrm{~g} \mathrm{~kg}^{-1} \mathrm{seed}$ (Seed treatment before sowing) + Biophos $12.5 \mathrm{~kg} \mathrm{ha}^{-1}$ (Broadcasting) Biophos $5 \mathrm{~g} \mathrm{~kg} \mathrm{k}^{-1}$ seed (Seed treatment before sowing) + Biophos $12.5 \mathrm{~kg} \mathrm{~h}^{-1}$ (Broadcasting), Biophos $5 \mathrm{~g} \mathrm{~kg}-1$ seed (Seed treatment before sowing) + Biophos $25.0 \mathrm{~kg} \mathrm{ha}^{-1}$ (Broadcasting), Biophos $25.0 \mathrm{~kg} \mathrm{ha}^{-1}$ (Broadcasting), Biophos $12.5 \mathrm{~kg} \mathrm{ha}^{-1}$ (Broadcasting), Biophos $10 \mathrm{~g} \mathrm{~kg}^{-1}$ seed (Seed treatment before sowing) and Biophos $5 \mathrm{~g}$ $\mathrm{kg}^{-1}$ seed (Seed treatment before sowing) ranked 2nd, 3rd, 4th, 5th, 6th and 7th in all the traits particularly fodder yield $\left(38.27,31.93,29.73,23.10,20.43,17.70\right.$ and $\left.15.33 \mathrm{t} \mathrm{ha}^{-1}\right)$, respectively. However, minimum $\left(30.33 \mathrm{~m}^{-2}\right)$ seed germination, $115.60 \mathrm{~cm}$ plant height, leaves plant ${ }^{-1}(6.53)$, nodes plant $^{-1}(2.13)$, stem girth $(2.38 \mathrm{~cm})$, fodder weight $\left(1.06 \mathrm{~kg} \mathrm{~m}^{-2}\right)$ and fodder yield $\left(10.60 \mathrm{t} \mathrm{ha}^{-1}\right)$ were recorded in No Biophos (Control). Hence, results concluded that combined application of Biophos 10 $\mathrm{g} \mathrm{kg}^{-1}$ seed (Seed treatment before sowing) + Biophos $25.0 \mathrm{~kg} \mathrm{~h}^{-1}$ (Broadcasting) was found most suitable which produced maximum values for almost all the parameters studied, particularly fodder yield (41.33 tha-1).

Keywords: Biofertilizers; Biophos; Maize; Seed germination; Yield 


\section{Introduction}

Maize (Zea mays L.) is the most important food and feed crop. In Pakistan, maize is the third most important cereal after wheat and rice, being grown on about one million hectares. Maize is a crop plant characterized by a high potential of biomass. Maize makes high quality silage for dairy cattle, beef and sheep at less cost than silage made from grass. Many research workers have reported that maize silage in the diet of dairy cows increases forage intake, milk yield and milk protein content [1]. Green fodder is the most valuable and cheapest source of feed for the livestock. It is very rich source of energy, nutrient elements, carbohydrates, proteins and water. Fodder shortage is the major limiting factor for livestock Production [2].

Application of biological fertilizer mycorrhiza had a significant effect on plant height, flag leaf width, grains ear $^{-1}$ and flag leaf length of maize. Interaction effects of mycorrhiza $\times$ varieties were non- significant plant height [3]. Maize quantity increased by utilization of bio-phosphate fertilizer [4]. The quantitative characteristics were significantly increased by phosphate-solution microorganisms [5]. A combination of mycorrhiza and bacteria holds promise for the organic cropping system of maize. Therefore, in organic and low input cropping systems, a combination of mycorrhiza and free-living bacteria performed satisfactorily [6]. The growth and yield traits of maize in compounds phosphate-solubilising bacteria and Mycorrhiza fungi treatment were higher than other treatments under drought stress conditions except root colonization. Accordingly, this experiment showed that phosphate solubilising micro-organisms can positively have effect on the increase of plant growth and phosphorus absorption in maize plant, leading to plant tolerance improving under drought stress conditions [7]. Yosefi et al. [8] suggested that maximum biological yield and grain weight of maize was obtained by inoculation of seeds with biological phosphate fertilizer prior to sowing. The findings of a study showed that the effect of plant growth promoting rhizobacteria on all traits was significant. Double-inoculation of Azotobacter and Azospirillum had the highest plant height $(212.4 \mathrm{~cm})$, stem diameter $(2.5$ $\mathrm{cm})$, rows ear $^{-1}$ (14.5 row), grains row $^{-1}$ (44.2), 1000-grain weight (315.4 g), grain yield (10190 kg ha-1), biological yield (21320 $\left.\mathrm{kg} \mathrm{ha}^{-1}\right)$ and protein content $(10.7 \%)$ when compared with other treatments. In view of above facts, the study was proposed to evaluate the impact of different doses and application methods of biophos (biofertilizer) on growth and fodder yield of maize.

\section{Materials and Methods}

The field study to evaluate the impact of different doses and application methods of biophos (biofertilizer) on growth and fodder yield of maizewas carried out at Students Farm, Department of Agronomy, Faculty of Crop Production, Sindh Agriculture University, Tandojam during Kharif, 2013. The experiment was laid out in a three replicated randomized complete block design (RCBD), having net plot size $5 \mathrm{~m} \mathrm{x} 4 \mathrm{~m}$ (20 $\mathrm{m}^{2}$ ). The treatments included: No Biophos (Control), Biophos $5 \mathrm{~g} \mathrm{~kg}^{-1}$ seed (Seed treatment before sowing), Biophos $10 \mathrm{~g} \mathrm{~kg}^{-1}$ seed (Seed treatment before sowing), Biophos $12.5 \mathrm{~kg} \mathrm{~h}^{-1}$ (Broadcasting), Biophos $25.0 \mathrm{~kg} \mathrm{~h}^{-1}$ (Broadcasting), Biophos $5 \mathrm{~g} \mathrm{~kg}^{-1}$ seed (Seed treatment before sowing) + Biophos $12.5 \mathrm{~kg} \mathrm{~h}^{-1}$ (Broadcasting), Biophos $5 \mathrm{~g} \mathrm{~kg}^{-1}$ seed (Seed treatment before sowing) + Biophos $25.0 \mathrm{~kg} \mathrm{~h}^{-1}$ (Broadcasting), Biophos $10 \mathrm{~g} \mathrm{~kg}^{-1}$ seed (Seed treatment before sowing) + Biophos $12.5 \mathrm{~kg} \mathrm{~h}^{-1}$ (Broadcasting) and Biophos $10 \mathrm{~g} \mathrm{~kg}^{-1}$ seed (Seed treatment before sowing) + Biophos $25.0 \mathrm{~kg} \mathrm{~h}^{-1}$ (Broadcasting). The observations were recorded on parameters of economic importance such as seed germination $\left(\mathrm{m}^{-2}\right)$, plant height $(\mathrm{cm})$, leaves plant ${ }^{-1}$, nodes plant ${ }^{-1}$, stem girth $(\mathrm{cm})$, fodder weight $\left(\mathrm{kg} \mathrm{m}^{-2}\right)$ and 
fodder yield $\left(\mathrm{t} \mathrm{ha}^{-1}\right)$. The collected data were statistically analyzed using Statistix 8.1 computer software (Statistix, 2006). The LSD test was applied to compare treatment means, where necessary.

\section{Results and Discussion}

\section{Seed germination $\left(\mathrm{m}^{-2}\right)$}

The results showed in (Table 1) that application of Biophos $10 \mathrm{~g} \mathrm{~kg}^{-1}$ seed (Seed treatment before sowing) + Biophos $25.0 \mathrm{~kg}$ $\mathrm{h}^{-1}$ (Broadcasting) produced maximum seed germination of $33.0 \mathrm{~m}^{-2}$, closely followed by $32.67,32.33$ and $32.00 \mathrm{~m}^{-2}$ recorded from the plots fertilized with Biophos $10 \mathrm{~g} \mathrm{~kg}^{-1}$ seed (Seed treatment before sowing) + Biophos
$12.5 \mathrm{~kg} \mathrm{~h}^{-1}$ (Broadcasting), Biophos $5 \mathrm{~g} \mathrm{~kg}^{-1}$ seed (Seed treatment before sowing) + Biophos $12.5 \mathrm{~kg} \mathrm{~h}^{-1}$ (Broadcasting) and Biophos $5 \mathrm{~g} \mathrm{~kg}^{-1}$ seed (Seed treatment before sowing) + Biophos $25.0 \mathrm{~kg} \mathrm{~h}^{-1}$ (Broadcasting), respectively. The seed germination was nominally reduced to 31.67 , 31.33, 31.00 and $30.67 \mathrm{~m}^{-2}$ in plots fertilized with Biophos $25.0 \mathrm{~kg} \mathrm{~h}^{-1}$ (Broadcasting), Biophos $12.5 \mathrm{~kg} \mathrm{~h}^{-1}$ (Broadcasting), Biophos $10 \mathrm{~g} \mathrm{~kg}^{-1}$ seed (Seed treatment before sowing) and Biophos $5 \mathrm{~g} \mathrm{~kg}^{-1}$ seed (Seed treatment before sowing), respectively. However, No Biophos (Control) had minimum seed germination of $30.33 \mathrm{~m}^{-2}$.

Table 1. Seed germination $\left(\mathrm{m}^{-2}\right)$ of maize as affected by doses and application methods of Biophos (Biofertilizer)

\begin{tabular}{|c|c|c|c|c|}
\hline Treatments & R-I & R-II & R-III & Mean \\
\hline No Biophos (Control) & 31.00 & 31.00 & 29.00 & 30.33 \\
\hline Biophos $5 \mathrm{~g} \mathrm{~kg}^{-1}$ seed (Seed treatment before sowing) & 31.00 & 31.00 & 30.00 & 30.67 \\
\hline Biophos $10 \mathrm{~g} \mathrm{~kg}^{-1}$ seed (Seed treatment $\quad$ before sowing) & 31.00 & 31.00 & 31.00 & 31.00 \\
\hline Biophos $12.5 \mathrm{~kg} \mathrm{~h}^{-1}$ (Broadcasting) & 31.00 & 32.00 & 31.00 & 31.33 \\
\hline Biophos $25.0 \mathrm{~kg} \mathrm{~h}^{-1}$ (Broadcasting) & 31.00 & 31.00 & 33.00 & 31.67 \\
\hline $\begin{array}{c}\text { Biophos } 5 \mathrm{~g} \mathrm{~kg}^{-1} \text { seed (Seed treatment before sowing) }+ \\
\text { Biophos } 12.5 \mathrm{~kg} \mathrm{~h}^{-1} \quad \text { (Broadcasting) }\end{array}$ & 32.00 & 33.00 & 32.00 & 32.33 \\
\hline $\begin{array}{c}\text { Biophos } 5 \mathrm{~g} \mathrm{~kg}^{-1} \text { seed (Seed treatment before sowing) }+ \\
\text { Biophos } 25.0 \mathrm{~kg} \mathrm{~h}^{-1} \quad \text { (Broadcasting) }\end{array}$ & 32.00 & 32.00 & 32.00 & 32.00 \\
\hline $\begin{array}{c}\text { Biophos } 10 \mathrm{~g} \mathrm{~kg}^{-1} \text { seed (Seed treatment before sowing) }+ \\
\text { Biophos } 12.5 \mathrm{~kg} \mathrm{~h}^{-1} \quad \text { (Broadcasting) }\end{array}$ & 33.00 & 32.00 & 33.00 & 32.67 \\
\hline $\begin{array}{c}\text { Biophos } 10 \mathrm{~g} \mathrm{~kg}^{-1} \text { seed (Seed treatment before sowing) }+ \\
\text { Biophos } 25.0 \mathrm{~kg} \mathrm{~h}^{-1} \quad \text { (Broadcasting) }\end{array}$ & 33.00 & 33.00 & 33.00 & 33.00 \\
\hline
\end{tabular}

\section{Plant height (cm)}

The data showed in (Table 2) that application of Biophos $10 \mathrm{~g} \mathrm{~kg}^{-1}$ seed (Seed treatment before sowing) + Biophos $25.0 \mathrm{~kg} \mathrm{~h}^{-1}$ (Broadcasting) produced maximum plant height of $178.87 \mathrm{~cm}$, closely followed by $169.53,157.13$ and $149.60 \mathrm{~cm}$ recorded from the plots fertilized with Biophos $10 \mathrm{~g} \mathrm{~kg}^{-1}$ seed (Seed treatment before sowing) + Biophos $12.5 \mathrm{~kg} \mathrm{~h}^{-1}$ (Broadcasting), Biophos $5 \mathrm{~g} \mathrm{~kg}^{-1}$ seed (Seed treatment before sowing) + Biophos $12.5 \mathrm{~kg} \mathrm{~h}^{-1}$ (Broadcasting) and
Biophos $5 \mathrm{~g} \mathrm{~kg}^{-1}$ seed (Seed treatment before sowing) + Biophos $25.0 \mathrm{~kg} \mathrm{~h}^{-1}$ (Broadcasting), respectively. The plant height was nominally reduced to 140.13 , 133.67, 131.53 and $129.20 \mathrm{~cm}$ in plots fertilized with Biophos $25.0 \mathrm{~kg} \mathrm{~h} \mathrm{~h}^{-1}$ (Broadcasting), Biophos $12.5 \quad \mathrm{~kg} \quad \mathrm{~h}^{-1}$ (Broadcasting), Biophos $10 \mathrm{~g} \mathrm{~kg}^{-1}$ seed (Seed treatment before sowing) and Biophos $5 \mathrm{~g} \mathrm{~kg}^{-}$ 1 seed (Seed treatment before sowing), respectively. However, No Biophos (Control) had minimum plant height of $115.60 \mathrm{~cm}$. 
Table 2. Plant height (cm) of maize as affected by doses and application methods of Biophos (Biofertilizer)

\begin{tabular}{|c|c|c|c|c|}
\hline Treatments & R-I & R-II & R-III & Mean \\
\hline No Biophos (Control) & 116.00 & 115.00 & 115.80 & 115.60 \\
\hline Biophos $5 \mathrm{~g} \mathrm{~kg}^{-1}$ seed (Seed treatment before sowing) & 129.60 & 128.60 & 129.40 & 129.20 \\
\hline Biophos $10 \mathrm{~g} \mathrm{~kg}^{-1}$ seed (Seed treatment & 145.40 & 124.20 & 125.00 & 131.53 \\
\hline Biophos $12.5 \mathrm{~kg} \mathrm{~h}^{-1}$ (Broadcasting) & 134.20 & 133.00 & 133.80 & 133.67 \\
\hline Biophos $25.0 \mathrm{~kg} \mathrm{~h}^{-1}$ (Broadcasting) & 140.80 & 139.40 & 140.20 & 140.13 \\
\hline $\begin{array}{c}\text { Biophos } 5 \mathrm{~g} \mathrm{~kg}^{-1} \text { seed (Seed treatment before sowing) }+ \\
\text { Biophos } 12.5 \mathrm{~kg} \mathrm{~h}^{-1} \text { (Broadcasting) }\end{array}$ & 150.00 & 149.00 & 149.80 & 149.60 \\
\hline $\begin{array}{c}\text { Biophos } 5 \mathrm{~g} \mathrm{~kg}^{-1} \text { seed (Seed treatment before sowing) }+ \\
\text { Biophos } 25.0 \mathrm{~kg} \mathrm{~h}^{-1} \quad \text { (Broadcasting) }\end{array}$ & 157.60 & 156.40 & 157.40 & 157.13 \\
\hline $\begin{array}{c}\text { Biophos } 10 \mathrm{~g} \mathrm{~kg}^{-1} \text { seed (Seed treatment before sowing) }+ \\
\text { Biophos } 12.5 \mathrm{~kg} \mathrm{~h}^{-1} \quad \text { (Broadcasting) }\end{array}$ & 170.00 & 168.80 & 169.80 & 169.53 \\
\hline $\begin{array}{c}\text { Biophos } 10 \mathrm{~g} \mathrm{~kg}^{-1} \text { seed (Seed treatment before sowing) }+ \\
\text { Biophos } 25.0 \mathrm{~kg} \mathrm{~h}^{-1} \quad \text { (Broadcasting) }\end{array}$ & 179.20 & 178.20 & 179.20 & 178.87 \\
\hline
\end{tabular}

\section{Leaves plant ${ }^{-1}$}

The results showed in (Table 3) that application ofBiophos $10 \mathrm{~g} \mathrm{~kg}^{-1}$ seed (Seed treatment before sowing) + Biophos 25.0 $\mathrm{kg} \mathrm{h}^{-1}$ (Broadcasting)produced maximum leaves plant ${ }^{-1}$ of 14.80 , closely followed by $13.80,12.80$ and 11.20 leaves plant ${ }^{-1}$ recorded from the plots fertilized with Biophos $10 \mathrm{~g}$ $\mathrm{kg}^{-1}$ seed (Seed treatment before sowing) + Biophos $12.5 \mathrm{~kg} \mathrm{~h}^{-1}$ (Broadcasting), Biophos $5 \mathrm{~g} \mathrm{~kg}^{-1}$ seed (Seed treatment before sowing) + Biophos $12.5 \mathrm{~kg} \mathrm{~h}^{-1}$ (Broadcasting) and
Biophos $5 \mathrm{~g} \mathrm{~kg}^{-1}$ seed (Seed treatment before sowing) + Biophos $25.0 \mathrm{~kg} \mathrm{~h} \mathrm{~h}^{-1}$ (Broadcasting), respectively. The leaves plant $^{-1}$ were nominally reduced to $9.80,9.00$, 7.80 and 7.00 in plots fertilized with Biophos $25.0 \mathrm{~kg} \mathrm{~h}^{-1}$ (Broadcasting), Biophos $12.5 \mathrm{~kg} \mathrm{~h}^{-}$ ${ }^{1}$ (Broadcasting), Biophos $10 \mathrm{~g} \mathrm{~kg}^{-1}$ seed (Seed treatment before sowing) and Biophos $5 \mathrm{~g} \mathrm{~kg}^{-1}$ seed (Seed treatment before sowing), respectively. However, No Biophos (Control) had minimum leaves plant ${ }^{-1}$ of $6.53 \mathrm{~m}^{-2}$.

Table 3. Leaves plant ${ }^{-1}$ of maize as affected by doses and application methods of Biophos (Biofertilizer)

\begin{tabular}{|c|c|c|c|c|}
\hline Treatments & R-I & R-II & R-III & Mean \\
\hline No Biophos (Control) & 6.60 & 6.40 & 6.60 & 6.53 \\
\hline Biophos $5 \mathrm{~g} \mathrm{~kg}^{-1}$ seed (Seed treatment & 7.20 & 6.80 & 7.00 & 7.00 \\
\hline Biophos $10 \mathrm{~g} \mathrm{~kg}^{-1}$ seed (Seed treatment before sowing) & 8.00 & 7.60 & 7.80 & 7.80 \\
\hline Biophos $12.5 \mathrm{~kg} \mathrm{~h}^{-1}$ (Broadcasting) & 9.20 & 8.80 & 9.00 & 9.00 \\
\hline Biophos $25.0 \mathrm{~kg} \mathrm{~h}^{-1}$ (Broadcasting) & 10.00 & 9.60 & 9.80 & 9.80 \\
\hline $\begin{array}{c}\text { Biophos } 5 \mathrm{~g} \mathrm{~kg}^{-1} \text { seed (Seed treatment before sowing) }+ \\
\text { Biophos } 12.5 \mathrm{~kg} \mathrm{~h}^{-1} \quad \text { (Broadcasting) }\end{array}$ & 11.40 & 11.00 & 11.20 & 11.20 \\
\hline $\begin{array}{c}\text { Biophos } 5 \mathrm{~g} \mathrm{~kg}^{-1} \text { seed (Seed treatment before sowing) }+ \\
\text { Biophos } 25.0 \mathrm{~kg} \mathrm{~h}^{-1} \quad \text { (Broadcasting) }\end{array}$ & 13.00 & 12.60 & 12.80 & 12.80 \\
\hline $\begin{array}{c}\text { Biophos } 10 \mathrm{~g} \mathrm{~kg}^{-1} \text { seed (Seed treatment before sowing) }+ \\
\text { Biophos } 12.5 \mathrm{~kg} \mathrm{~h}^{-1} \quad \text { (Broadcasting) }\end{array}$ & 14.00 & 13.60 & 13.80 & 13.80 \\
\hline $\begin{array}{c}\text { Biophos } 10 \mathrm{~g} \mathrm{~kg}^{-1} \text { seed (Seed treatment before sowing) }+ \\
\text { Biophos } 25.0 \mathrm{~kg} \mathrm{~h}^{-1} \text { (Broadcasting) }\end{array}$ & 15.00 & 14.60 & 14.80 & 14.80 \\
\hline
\end{tabular}




\section{Nodes plant $\mathbf{t}^{-1}$}

The data showed in (Table 4) that application ofBiophos $10 \mathrm{~g} \mathrm{~kg}^{-1}$ seed (Seed treatment before sowing) + Biophos $25.0 \mathrm{~kg} \mathrm{~h}^{-1}$ (Broadcasting)produced maximum nodes plant $^{-1}$ of 5.40 , closely followed by $4.53,4.13$ and 3.73 nodes plant $^{-1}$ recorded from the plots fertilized with Biophos $10 \mathrm{~g} \mathrm{~kg}^{-1}$ seed (Seed treatment before sowing) + Biophos $12.5 \mathrm{~kg} \mathrm{~h}^{-1}$ (Broadcasting), Biophos $5 \mathrm{~g} \mathrm{~kg}^{-1}$ seed (Seed treatment before sowing) + Biophos $12.5 \mathrm{~kg} \mathrm{~h}^{-1}$ (Broadcasting) and
Biophos $5 \mathrm{~g} \mathrm{~kg}^{-1}$ seed (Seed treatment before sowing) + Biophos $25.0 \mathrm{~kg} \mathrm{~h}^{-1}$ (Broadcasting), respectively. The number nodes plant ${ }^{-1}$ was nominally reduced to 3.33 , $3.00,2.60$ and 2.33 in plots fertilized with Biophos $25.0 \mathrm{~kg} \mathrm{~h}^{-1}$ (Broadcasting), Biophos $12.5 \mathrm{~kg} \mathrm{~h}^{-1}$ (Broadcasting), Biophos $10 \mathrm{~g} \mathrm{~kg}^{-1}$ seed (Seed treatment before sowing) and Biophos $5 \mathrm{~g} \mathrm{~kg}^{-1}$ seed (Seed treatment before sowing), respectively. However, No Biophos (Control) had minimum nodes plant ${ }^{-1}$ of 2.13.

Table 4. Nodes plant ${ }^{-1}$ of maize as affected by doses and application methods of Biophos (Biofertilizer)

\begin{tabular}{|c|c|c|c|c|}
\hline Treatments & R-I & R-II & R-III & Mean \\
\hline No Biophos (Control) & 2.20 & 2.00 & 2.20 & 2.13 \\
\hline Biophos $5 \mathrm{~g} \mathrm{~kg}^{-1}$ seed (Seed treatment before sowing) & 2.40 & 2.20 & 2.40 & 2.33 \\
\hline Biophos $10 \mathrm{~g} \mathrm{~kg}^{-1}$ seed (Seed treatment & 2.60 & 2.40 & 2.80 & 2.60 \\
\hline Biophos $12.5 \mathrm{~kg} \mathrm{~h}^{-1}$ (Broadcasting) & 3.00 & 2.80 & 3.20 & 3.00 \\
\hline Biophos $25.0 \mathrm{~kg} \mathrm{~h}^{-1}$ (Broadcasting) & 3.40 & 3.20 & 3.40 & 3.33 \\
\hline $\begin{array}{c}\text { Biophos } 5 \mathrm{~g} \mathrm{~kg}^{-1} \text { seed (Seed treatment before sowing) }+ \\
\text { Biophos } 12.5 \mathrm{~kg} \mathrm{~h}^{-1} \text { (Broadcasting) }\end{array}$ & 3.80 & 3.60 & 3.80 & 3.73 \\
\hline $\begin{array}{c}\text { Biophos } 5 \mathrm{~g} \mathrm{~kg}^{-1} \text { seed (Seed treatment before sowing) }+ \\
\text { Biophos } 25.0 \mathrm{~kg} \mathrm{~h}^{-1} \text { (Broadcasting) }\end{array}$ & 4.20 & 4.00 & 4.20 & 4.13 \\
\hline $\begin{array}{c}\text { Biophos } 10 \mathrm{~g} \mathrm{~kg}^{-1} \text { seed (Seed treatment before sowing) }+ \\
\text { Biophos } 12.5 \mathrm{~kg} \mathrm{~h}^{-1} \quad \text { (Broadcasting) }\end{array}$ & 4.60 & 4.40 & 4.60 & 4.53 \\
\hline $\begin{array}{c}\text { Biophos } 10 \mathrm{~g} \mathrm{~kg}^{-1} \text { seed (Seed treatment before sowing) }+ \\
\text { Biophos } 25.0 \mathrm{~kg} \mathrm{~h}^{-1} \quad \text { (Broadcasting) }\end{array}$ & 5.40 & 5.20 & 5.60 & 5.40 \\
\hline
\end{tabular}

\section{Stem girth (cm)}

The results showed in (Table 5) that application ofBiophos $10 \mathrm{~g} \mathrm{~kg}^{-1}$ seed (Seed treatment before sowing) + Biophos 25.0 $\mathrm{kg} \mathrm{h}^{-1}$ (Broadcasting)resulted in maximum stem girth of $4.75 \mathrm{~cm}$, closely followed by $4.43,4.31$ and $4.13 \mathrm{~cm}$ stem girth recorded from the plots fertilized with Biophos $10 \mathrm{~g}$ $\mathrm{kg}^{-1}$ seed (Seed treatment before sowing) + Biophos $12.5 \mathrm{~kg} \mathrm{~h}^{-1}$ (Broadcasting), Biophos $5 \mathrm{~g} \mathrm{~kg}^{-1}$ seed (Seed treatment before sowing) + Biophos $12.5 \mathrm{~kg} \mathrm{~h}^{-1}$ (Broadcasting) and Biophos $5 \mathrm{~g} \mathrm{~kg}^{-1}$ seed (Seed treatment before sowing) + Biophos $25.0 \mathrm{~kg} \quad \mathrm{~h}^{-1}$ (Broadcasting), respectively. The stem girth was nominally reduced to $3.95,3.67,3.25$ and $2.93 \mathrm{~cm}$ in plots fertilized with Biophos
$25.0 \mathrm{~kg} \mathrm{~h}^{-1}$ (Broadcasting), Biophos $12.5 \mathrm{~kg} \mathrm{~h}^{-}$

1 (Broadcasting), Biophos $10 \mathrm{~g} \mathrm{~kg}^{-1}$ seed (Seed treatment before sowing) and Biophos $5 \mathrm{~g} \mathrm{~kg}^{-1}$ seed (Seed treatment before sowing), respectively. However, No Biophos (Control) had minimum stem girth of $2.38 \mathrm{~cm}$.

\section{Fodder weight $\left(\mathrm{kg} \mathrm{m}^{-2}\right)$}

The results showed in (Table 6) that application ofBiophos $10 \mathrm{~g} \mathrm{~kg}^{-1}$ seed (Seed treatment before sowing) + Biophos $25.0 \mathrm{~kg}$ $\mathrm{h}^{-1}$ (Broadcasting)produced maximum fodder weight $\left(\mathrm{m}^{-2}\right)$ of $4.13 \mathrm{~kg}$, closely followed by $3.83,3.19$ and $2.97 \mathrm{~kg}$ fodder weight $\left(\mathrm{m}^{-2}\right)$ recorded from the plots fertilized with Biophos $10 \mathrm{~g} \mathrm{~kg}^{-1}$ seed (Seed treatment before sowing) + Biophos $12.5 \mathrm{~kg} \mathrm{~h}^{-1}$ (Broadcasting), Biophos $5 \mathrm{~g} \mathrm{~kg}^{-1}$ seed (Seed 
treatment before sowing) + Biophos $12.5 \mathrm{~kg}$ $\mathrm{h}^{-1}$ (Broadcasting) and Biophos $5 \mathrm{~g} \mathrm{~kg}^{-1}$ seed (Seed treatment before sowing) + Biophos $25.0 \mathrm{~kg} \mathrm{~h}^{-1}$ (Broadcasting), respectively. The fodder weight $\left(\mathrm{m}^{-2}\right)$ was nominally reduced to $2.31,2.04,1.77$ and $1.53 \mathrm{~kg}$ in plots fertilized with Biophos $25.0 \quad \mathrm{~kg} \quad \mathrm{~h}^{-1}$
(Broadcasting), Biophos $12.5 \quad \mathrm{~kg} \quad \mathrm{~h}^{-1}$ (Broadcasting), Biophos $10 \mathrm{~g} \mathrm{~kg}^{-1}$ seed (Seed treatment before sowing) and Biophos $5 \mathrm{~g} \mathrm{~kg}^{-}$ 1 seed (Seed treatment before sowing), respectively. However, No Biophos (Control) had minimum fodder weight $\left(\mathrm{m}^{-2}\right)$ of $1.06 \mathrm{~kg}$.

Table 5. Stem girth (cm) of maize as affected by doses and application methods of Biophos (Biofertilizer)

\begin{tabular}{|c|c|c|c|c|}
\hline Treatments & R-I & R-II & R-III & Mean \\
\hline No Biophos (Control) & 2.40 & 2.36 & 2.38 & 2.38 \\
\hline Biophos $5 \mathrm{~g} \mathrm{~kg}^{-1}$ seed (Seed treatment before sowing) & 2.96 & 2.90 & 2.92 & 2.93 \\
\hline Biophos $10 \mathrm{~g} \mathrm{~kg}^{-1}$ seed (Seed treatment before sowing) & 3.28 & 3.22 & 3.24 & 3.25 \\
\hline Biophos $12.5 \mathrm{~kg} \mathrm{~h}^{-1}$ (Broadcasting) & 3.70 & 3.64 & 3.66 & 3.67 \\
\hline Biophos $25.0 \mathrm{~kg} \mathrm{~h}^{-1}$ (Broadcasting) & 3.98 & 3.92 & 3.94 & 3.95 \\
\hline $\begin{array}{c}\text { Biophos } 5 \mathrm{~g} \mathrm{~kg}^{-1} \text { seed (Seed treatment before sowing) }+ \\
\text { Biophos } 12.5 \mathrm{~kg} \mathrm{~h}^{-1} \quad \text { (Broadcasting) }\end{array}$ & 4.16 & 4.10 & 4.12 & 4.13 \\
\hline $\begin{array}{c}\text { Biophos } 5 \mathrm{~g} \mathrm{~kg}^{-1} \text { seed (Seed treatment before sowing) + } \\
\text { Biophos } 25.0 \mathrm{~kg} \mathrm{~h}^{-1} \quad \text { (Broadcasting) }\end{array}$ & 4.34 & 4.28 & 4.30 & 4.31 \\
\hline $\begin{array}{c}\text { Biophos } 10 \mathrm{~g} \mathrm{~kg}^{-1} \text { seed (Seed treatment before sowing) }+ \\
\text { Biophos } 12.5 \mathrm{~kg} \mathrm{~h}^{-1} \quad \text { (Broadcasting) }\end{array}$ & 4.46 & 4.40 & 4.42 & 4.43 \\
\hline $\begin{array}{c}\text { Biophos } 10 \mathrm{~g} \mathrm{~kg}^{-1} \text { seed (Seed treatment before sowing) }+ \\
\text { Biophos } 25.0 \mathrm{~kg} \mathrm{~h}^{-1} \quad \text { (Broadcasting) }\end{array}$ & 4.78 & 4.74 & 4.74 & 4.75 \\
\hline
\end{tabular}

Table 6. Fodder weight $\left(\mathrm{kg} \mathrm{m}^{-2}\right)$ of maize as affected by doses and application methods of Biophos (Biofertilizer)

\begin{tabular}{|c|c|c|c|c|}
\hline Treatments & R-I & R-II & R-III & Mean \\
\hline No Biophos (Control) & 1.02 & 1.07 & 1.09 & 1.06 \\
\hline Biophos $5 \mathrm{~g} \mathrm{~kg}^{-1}$ seed (Seed treatment before sowing) & 1.50 & 1.54 & 1.56 & 1.53 \\
\hline Biophos $10 \mathrm{~g} \mathrm{~kg}^{-1}$ seed (Seed treatment before sowing) & 1.77 & 1.78 & 1.76 & 1.77 \\
\hline Biophos $12.5 \mathrm{~kg} \mathrm{~h}^{-1}$ (Broadcasting) & 2.04 & 2.02 & 2.07 & 2.04 \\
\hline Biophos $25.0 \mathrm{~kg} \mathrm{~h}^{-1}$ (Broadcasting) & 2.28 & 2.31 & 2.34 & 2.31 \\
\hline $\begin{array}{c}\text { Biophos } 5 \mathrm{~g} \mathrm{~kg}^{-1} \text { seed (Seed treatment before sowing) }+ \\
\text { Biophos } 12.5 \mathrm{~kg} \mathrm{~h}^{-1} \quad \text { (Broadcasting) }\end{array}$ & 2.97 & 2.94 & 3.01 & 2.97 \\
\hline $\begin{array}{c}\text { Biophos } 5 \mathrm{~g} \mathrm{~kg}^{-1} \text { seed (Seed treatment before sowing) }+ \\
\text { Biophos } 25.0 \mathrm{~kg} \mathrm{~h}^{-1} \quad \text { (Broadcasting) }\end{array}$ & 3.15 & 3.21 & 3.22 & 3.19 \\
\hline $\begin{array}{c}\text { Biophos } 10 \mathrm{~g} \mathrm{~kg}^{-1} \text { seed (Seed treatment before sowing) + } \\
\text { Biophos } 12.5 \mathrm{~kg} \mathrm{~h}^{-1} \quad \text { (Broadcasting) }\end{array}$ & 3.80 & 3.83 & 3.85 & 3.83 \\
\hline $\begin{array}{c}\text { Biophos } 10 \mathrm{~g} \mathrm{~kg}^{-1} \text { seed (Seed treatment before sowing) + } \\
\text { Biophos } 25.0 \mathrm{~kg} \mathrm{~h}^{-1} \quad \text { (Broadcasting) }\end{array}$ & 4.08 & 4.12 & 4.20 & 4.13 \\
\hline
\end{tabular}

\section{Fodder yield ( ha $^{-1}$ )}

The results showed in (Table 7) that application of Biophos $10 \mathrm{~g} \mathrm{~kg}^{-1}$ seed (Seed treatment before sowing) + Biophos $25.0 \mathrm{~kg}$ $\mathrm{h}^{-1}$ (Broadcasting)produced maximum fodder yield of $41.33 \mathrm{t} \mathrm{ha}^{-1}$, closely followed by
38.27, 31.93 and $29.73 \mathrm{t} \mathrm{ha}^{-1}$ fodder yield recorded from the plots fertilized with Biophos $10 \mathrm{~g} \mathrm{~kg}^{-1}$ seed (Seed treatment before sowing) + Biophos $12.5 \mathrm{~kg} \mathrm{~h}^{-1}$ (Broadcasting), Biophos $5 \mathrm{~g} \mathrm{~kg}^{-1}$ seed (Seed treatment before sowing) + Biophos $12.5 \mathrm{~kg}$ 
$\mathrm{h}^{-1}$ (Broadcasting) and Biophos $5 \mathrm{~g} \mathrm{~kg}^{-1}$ seed (Seed treatment before sowing) + Biophos $25.0 \mathrm{~kg} \mathrm{~h}^{-1}$ (Broadcasting), respectively. The fodder yield was nominally reduced to 23.10 , 20.43, 17.70 and $15.33 \mathrm{t} \mathrm{ha}^{-1}$ in plots fertilized with Biophos $25.0 \mathrm{~kg} \mathrm{~h}^{-1}$ (Broadcasting),
Biophos $12.5 \mathrm{~kg} \mathrm{~h}^{-1}$ (Broadcasting), Biophos $10 \mathrm{~g} \mathrm{~kg}^{-1}$ seed (Seed treatment before sowing) and Biophos $5 \mathrm{~g} \mathrm{~kg}^{-1}$ seed (Seed treatment before sowing), respectively. However, No Biophos (Control) had minimum fodder yield of $10.60 \mathrm{t} \mathrm{ha}^{-1}$.

Table 7. Fodder yield (kg ha-1) of maize as affected by doses and application methods of Biophos (Biofertilizer)

\begin{tabular}{|c|c|c|c|c|}
\hline Treatments & R-I & R-II & R-III & Mean \\
\hline No Biophos (Control) & 10.20 & 10.70 & 10.90 & 10.60 \\
\hline Biophos $5 \mathrm{~g} \mathrm{~kg}^{-1}$ seed (Seed treatment before sowing) & 15.00 & 15.40 & 15.60 & 15.33 \\
\hline Biophos $10 \mathrm{~g} \mathrm{~kg}^{-1}$ seed (Seed treatment before sowing) & 17.70 & 17.80 & 17.60 & 17.70 \\
\hline Biophos $12.5 \mathrm{~kg} \mathrm{~h}^{-1}$ (Broadcasting) & 20.40 & 20.20 & 20.70 & 20.43 \\
\hline Biophos $25.0 \mathrm{~kg} \mathrm{~h}^{-1}$ (Broadcasting) & 22.80 & 23.10 & 23.40 & 23.10 \\
\hline $\begin{array}{c}\text { Biophos } 5 \mathrm{~g} \mathrm{~kg}^{-1} \text { seed (Seed treatment before sowing) }+ \\
\text { Biophos } 12.5 \mathrm{~kg} \mathrm{~h}^{-1} \text { (Broadcasting) }\end{array}$ & 29.70 & 29.40 & 30.10 & 29.73 \\
\hline $\begin{array}{c}\text { Biophos } 5 \mathrm{~g} \mathrm{~kg}^{-1} \text { seed (Seed treatment before sowing) }+ \\
\text { Biophos } 25.0 \mathrm{~kg} \mathrm{~h}^{-1} \quad \text { (Broadcasting) }\end{array}$ & 31.50 & 32.10 & 32.20 & 31.93 \\
\hline $\begin{array}{c}\text { Biophos } 10 \mathrm{~g} \mathrm{~kg}^{-1} \text { seed (Seed treatment before sowing) }+ \\
\text { Biophos } 12.5 \mathrm{~kg} \mathrm{~h}^{-1} \quad \text { (Broadcasting) }\end{array}$ & 38.00 & 38.30 & 38.50 & 38.27 \\
\hline $\begin{array}{c}\text { Biophos } 10 \mathrm{~g} \mathrm{~kg}^{-1} \text { seed (Seed treatment before sowing) }+ \\
\text { Biophos } 25.0 \mathrm{~kg} \mathrm{~h}^{-1} \text { (Broadcasting) }\end{array}$ & 40.80 & 41.20 & 42.00 & 41.33 \\
\hline
\end{tabular}

\section{Discussion}

The positive effect of using biofertilizer could be attributed to increased water and nutrient uptake due to development and expansion of roots and also to biological nitrogen fixation by biofertilizers. The results are in agreement with those of [9] who reported root, shoot weight with dual inoculation in maize. Use of biofertilizers offers agronomic and environmental benefits for intensive agricultural systems. The results obtained in this study confirm the findings of [10] who stated that Seed inoculation with Rhizobium, phosphorus solubilizing bacteria, and organic amendment increased seed production of groundnut. The increasing growth and fodder yield of maize may be attributed to the plant growth promoting substances by root colonizing bacteria more than the biological nitrogen fixation. [11] stated that yield increased due to promoting root growth which in turn enhancing nutrients and water uptake from the soil. The results are at par with the findings of [12] who reported that significant increase in dry matter accumulation and leaf area index due to biofertilizers along with organic manure has a synergistic effect on subsequent plant growth and increase bacterial development, as Microbin and Rizobacterine inoculum cause biological N2 fixation, which improves plant growth. Plant height is the function of both genetic constitution and the environmental conditions under which it is grown. The greater plant height indicated maximum chances for radiation interception, its utilization efficiency and consequently higher photosynthetic rate. Leaves are the food manufacturing factories of the plant and the greater number of leaves ensured the increased fodder yield due to higher photosynthetic capacity by increasing LAI. The increase in growth attributes like plant height, leaves plant ${ }^{-}$ ${ }^{1}$, nodes plant ${ }^{-1}$ and stem girth $(\mathrm{cm})$, resultantly increased fodder yield $\left(\mathrm{t} \mathrm{ha}^{-1}\right)$ might be due to the improvement in translocation of assimilates. Positive effect of biofertilizer may 
be resulted from its ability to increase the availability of Phosphorus and other nutrients. Growth improvement and yield and biomass increasing was reported with the biofertilizer application which account for important benefit to the maize producers and maize fodder production, causing decrease in the inputs of production because of economizing much money to chemical fertilizers and increasing in biological yield in the production of the unit area, as well as its undamaged effect on the soil and environment, and on other hand its positive effect on physiology of growth performance [13]. Beneficial metabolites synthesis and plant growth promoters exudation are other azotobacter functions. Mycorrhizal symbiosis also improves plants growth through different mechanisms such as better uptake of water and nutrients, controlling stomata, root hydrolic conductivity, photosynthesis and increasing plants resistance to pathogens [14]. Jilani et al. [15]reported that energy efficiency, crop growth and yield may increase in organic farming compared with conventional systems. Mycorrhiza and bacteria with symbiosis activity could cause increase in phosphorus around roots of plants and absorption by roots.

\section{Conclusion}

The results concluded that seed germination, growth and fodder yield traits of maize variety Hycorn-8288were affected positively and significantly $(\mathrm{P}<0.05)$ by different doses and application methods of Biophos (Biofertilizer) as compared to No Biophos (Control). Combined application of Biophos $10 \mathrm{~g} \mathrm{~kg}^{-1}$ seed (Seed treatment before sowing) + Biophos $25.0 \mathrm{~kg} \mathrm{~h}^{-1}$ (Broadcasting) produced maximum values for almost all the parameters studied, particularly fodder yield (41.33 $\left.\mathrm{t} \mathrm{ha}^{-1}\right)$. Hence, the above results suggested that biofertilizers play very important role in solubilizing and making available mineral phosphorus in optimum concentration to plant roots, and enhancing crop growth and yield.

\section{Authors' contributions}

Conceived and designed the experiments: KA Brohi, FM Baloch \& AL Siyal, Performed the experiments: KA Brohi \& AH Sial, Analyzed the data: MA Sahito \& AL Siyal, Contributed materials/ analysis/ tools: KR Kalwar, BS Mengal \& AA Korejo, Wrote the paper: AL Siyal.

\section{References}

1. Ali S, Sahiba MM, Malik $\underline{A}-A$, Hassan $\underline{F}$ F \& Ansar M (M (2012)). Growth of rainfed fodder maize under different levels of nitrogen and phosphorus. PakJ Agric Res 25(3): 196-205.

2. Younas M \& Yaqoob $\mathrm{M}$ (M (2005)). Feed resources of livestock in Punjab, Pakistan. Livestock Res for Rural Devel 17:2.

3. Mobasser HR \& Abolghasem M (M (2012)). Mycorrhizal bio-fertilizer applications on yield seed corn varieties in Iran. Ann Biol Res 3(2): 1109-1116.

4. Ali S, Riaz AK, Ghazal M, Arif M, Fida M \& Saiqa B (2008). Assessment of different crop nutrient management practices for yield improvement. Aus $J$ Crop Sci 2(3): 150-157.

5. Siyal AL, Fozia KS \& Tahira J (2021). Yield from genetic variability of bread wheat (TriticumaestivumL.) genotypes under water stress condition: A case study of Tandojam, Sindh. Pure \& Appl Biol 10(3): 841-860.

6. Soleimanzadeh $\mathrm{H} \&$ Ghooshchi F (2013). Response of growth and yield of maize to biofertilizers in organic and conventional cropping systems. Inter $J$ of Agric and Crop Sci 5(7): 797-801.

7. Zarabi M, Alahdadi I, Akbari GA \& Akbari GA (2011). A study on the effects of different Biofertilizer combinations on yield, its components and growth indices of corn (Zea mays L.) under drought stress condition. African J Agric Res 6(3): 681-685. 
8. Yosefi K, Galavi M, Mahmod R \& Roholla MS (2011). Effect of biophosphate and chemical phosphorus fertilizer accompanied with micronutrient foliar application on growth, yield and yield components of maize (SC-704). Aust J of Crop Sci 5(2): 175-180.

9. Kouchebagh SB, Mirshekari B \& Farahvash F (2012). Improvement of corn yield by seed biofertilization and urea application. World Appl Sci J 16(9): 1239-1242.

10. Siyal AL (2017). Effect of bio fertilizer in addition with phosphorus on the growth of maize (Zea mayz L.). Intern J of $A d v$ Res 5(12): 527-532.

11. Mangrio GS, Altaf AS, Dahot MU \& Khaskheli AJ (2010). Growth and yield response of Zea mays to different treatments of biofertilizers. Pak $J$ Biotechnol 7(1-2): 13- 115.

12. Minapour A, Khourgami A, Rafiee M, Pezeshkpour P \& Nasrollahi H (2013). Study the effect of phosphate fertilizer (fertile2), phosphorus and foliar nitrogen on yield and yield components of maize cultivar SC 704 in weather conditions of Kouhdasht. Ann of Biol Res 4(5): 212-215.

13. Mohammed AA (2012). Effect of BioFertilizer on physiology of growth and development of maize (Zea mays L.) in Sulaimani region. Mesopotamia J Agric 40(1): 9-21.

14. Siyal AL, Ali GC, Nasiruddin S, Jay KS, Tahira J, Fozia KS \& Muhammad SC (2021). Screening of Wheat Genotypes for Morphological, Physiological and Phenological Traits Under Climatic Condition. Eur J of Biol and Biotechnol 2(2): 87-91.

15. Jilani G, Abida AA, Fauzia Y, Imranhaider S, Muhammad A \& Muhammad A (2007). Prospects of supplementing chemical fertilizers with organic and bio-fertilizers for crop production in rainfed conditions. Afri Crop Sci Conf Proc 8: 1513-1516. 